\title{
Mass-invariance of the iron enrichment in the hot haloes of massive ellipticals, groups, and clusters of galaxies
}

\author{
F. Mernier, ${ }^{1,2,3}$ J. de Plaa, ${ }^{3}$ N. Werner, ${ }^{1,4,5}$ J. S. Kaastra, ${ }^{3,6}$ A. J. J. Raassen, ${ }^{3}$ L. Gu, ${ }^{7}$ \\ J. Mao, ${ }^{3,6}$ I. Urdampilleta, ${ }^{3,6}$ N. Truong ${ }^{1}$ and A. Simionescu ${ }^{8}$ \\ ${ }^{1}$ MTA-Eötvös University Lendület Hot Universe Research Group, Pázmány Péter sétány 1/A, Budapest 1117, Hungary \\ ${ }^{2}$ Institute of Physics, Eötvös University, Pázmány Péter sétány 1/A, Budapest 1117, Hungary \\ ${ }^{3}$ SRON Netherlands Institute for Space Research, Sorbonnelaan 2, NL-3584 CA Utrecht, the Netherlands \\ ${ }^{4}$ Department of Theoretical Physics and Astrophysics, Faculty of Science, Masaryk University, Kotlárská 2, Brno, 611 37, Czech Republic \\ ${ }^{5}$ School of Science, Hiroshima University, 1-3-1 Kagamiyama, Higashi-Hiroshima 739-8526, Japan \\ ${ }^{6}$ Leiden Observatory, Leiden University, PO Box 9513, NL-2300 RA Leiden, the Netherlands \\ ${ }^{7}$ RIKEN Nishina Center, 2-1 Hirosawa, Wako, Saitama 351-0198, Japan \\ ${ }^{8}$ Institute of Space and Astronautical Science (ISAS), JAXA, 3-1-1 Yoshinodai, Chuo-ku, Sagamihara, Kanagawa 252-5210, Japan
}

Accepted 2018 May 2. Received 2018 May 02; in original form 2018 March 12

\begin{abstract}
$\mathrm{X}$-ray measurements find systematically lower $\mathrm{Fe}$ abundances in the X-ray emitting haloes pervading groups $(k T \lesssim 1.7 \mathrm{keV}$ ) than in clusters of galaxies. These results have been difficult to reconcile with theoretical predictions. However, models using incomplete atomic data or the assumption of isothermal plasmas may have biased the best-fitting Fe abundance in groups and giant elliptical galaxies low. In this work, we take advantage of a major update of the atomic code in the spectral fitting package SPEX to re-evaluate the Fe abundance in 43 clusters, groups, and elliptical galaxies (the CHEERS sample) in a self-consistent analysis and within a common radius of $0.1 r_{500}$. For the first time, we report a remarkably similar average Fe enrichment in all these systems. Unlike previous results, this strongly suggests that metals are synthesized and transported in these haloes with the same average efficiency across two orders of magnitude in total mass. We show that the previous metallicity measurements in low-temperature systems were biased low due to incomplete atomic data in the spectral fitting codes. The reasons for such a code-related Fe bias, also implying previously unconsidered biases in the emission measure and temperature structure, are discussed.
\end{abstract}

Key words: galaxies: clusters: intracluster medium-X-rays: galaxies: clusters-X-rays: galaxies.

\section{INTRODUCTION}

The largest gravitationally bound structures in the Universe, such as giant elliptical galaxies, groups, and clusters of galaxies, are pervaded by hot, X-ray emitting atmospheres, which typically account for an important fraction (up to $\sim 50-90$ per cent) of the total baryonic mass of these systems (e.g. Giodini et al. 2009). These hot atmospheres, hereafter defined for convenience as intracluster medium (ICM), are also rich in heavy elements that were produced by Type Ia and core-collapse supernovae within cluster/group members and giant central galaxies (for recent reviews, see Werner et al. 2008; de Plaa 2013; de Plaa \& Mernier 2017). Whereas observations and simulations suggest that metals in cluster outskirts were released more than 10 Gyr ago (e.g. Biffi et al. 2017, 2018; Urban

\footnotetext{
^E-mail: mernier@caesar.elte.hu
}

et al. 2017), the epoch and origin of the enrichment in the vicinity of central galaxies is less clear.

Because the ICM is in a collisional ionization equilibrium, abundances of various elements (typically from oxygen to nickel) can be robustly measured. This is especially true for Fe, whose both $\mathrm{K}$ - and L-shell transitions have high emissivities and fall within the typical energy windows $(\sim 0.5-10 \mathrm{keV})$ of our X-ray observatories. For this reason, Fe abundances can be precisely measured in the $\mathrm{X}$-ray haloes of both hot, massive clusters (via the $\mathrm{Fe}-\mathrm{K}$ transitions) and cooler, less massive groups and ellipticals (via the $\mathrm{Fe}-\mathrm{L}$ transitions). In turn, these $\mathrm{Fe}$ abundance measurements are usually interpreted as a reliable tracer of the overall metallicity in clusters and groups (e.g. de Plaa et al. 2017, and references therein), and are thus valuable to understand the history of metal enrichment in these systems.

In the past, several works extensively studied the Fe abundance in the hot gas of either nearby ellipticals and galaxy groups (e.g. 
Mahdavi et al. 2005; Finoguenov et al. 2006; Grange et al. 2011; Konami et al. 2014; Sasaki, Matsushita \& Sato 2014), or galaxy clusters (e.g. De Grandi \& Molendi 2001, 2009; Tamura et al. 2004; de Plaa et al. 2007; Matsushita 2011; Zhang et al. 2011). Very few studies, however, attempted to compare directly the metal content of all these systems together (e.g. Bregman, Anderson \& Dai 2010; Sun 2012).

In what has been perhaps the most comprehensive study so far, Yates, Thomas \& Henriques (2017) compiled from the literature a large number of $\mathrm{Fe}$ abundances measured in 79 nearby groups and clusters and homogenized these measurements by extrapolating them to a radius of $r_{500}$. While in hot clusters, the Fe abundance was found to converge to a rather uniform value of $\sim 0.3$ Solar, in lowtemperature groups and giant ellipticals the metallicity appeared to be on average significantly lower (see also Rasmussen \& Ponman $2007,2009)$. These results were not reproduced by predictions from semi-analytical models of galaxy evolution, in which (at least) as much Fe was expected in groups as in clusters (Yates et al. 2017).

Do theoretical models really miss some important chemodynamical process at play in galaxy groups, or do spectroscopic measurements instead suffer from unexpected biases in low-temperature systems? From an observational perspective, this question remains open. In fact, homogenizing Fe abundance measurements from the literature is very challenging, essentially because: (i) different authors utilized different data reduction and analysis methods, (ii) instrumental calibration and spectral models continuously evolved with years, and (iii) the lack of accurate measurements for radial Fe profiles of individual systems out to $r_{500}$ makes the extrapolation to this radius quite uncertain. Last but not least, cooler systems $(k T \lesssim$ $2 \mathrm{keV}$ ) require careful attention as the $\mathrm{Fe}-\mathrm{L}$ complex, which is unresolved by CCD instruments, may be underestimated if one assumes the plasma to be isothermal (the 'Fe-bias'; Buote \& Canizares 1994; Buote 2000). Since most of the baryons (and metals) are rather in groups than in clusters, determining their accurate, unbiased metallicity is nevertheless of a crucial importance to estimate the global metal budget of the Universe. Clearly, measurements of such metallicities in hot haloes at all masses need to be further investigated and better understood.

In a recent work (Mernier et al. 2016, hereafter Paper I), we used XMM-Newton EPIC observations to measure Fe - among other elemental abundances - in the hot haloes of 44 nearby coolcore ellipticals, groups, and clusters of galaxies (the CHEERS ${ }^{1}$ catalogue). Interestingly, we found an apparent deficit of Fe in the coolest systems, supporting the previous findings of Rasmussen \& Ponman $(2007,2009)$ and Yates et al. (2017), which are in tension with theoretical expectations. In that study, however, groups and ellipticals were investigated only within $0.05 r_{500}$, making it difficult to compare with most simulations given their limited resolution. In addition, a major update of the plasma models from the SPEX fitting package (Kaastra, Mewe \& Nieuwenhuijzen 1996) has been publicly released. As briefly noted in Mernier et al. (2017), such an improvement could affect the Fe abundance measured by CCD instruments in cooler plasmas and potentially revise our current picture of the ICM enrichment from massive ellipticals to the largest structures of the Universe.

In this Letter, we revisit the observed $\mathrm{Fe}$ abundances in the CHEERS sample by: (i) analyzing EPIC spectra within a common astrophysical radius of $0.1 r_{500}$ - easier to compare with simulations - and (ii) exploring how recent spectral model improvements alter the measured $\mathrm{Fe}$ abundances and their interpretation. Throughout this Letter, we assume $H_{0}=70 \mathrm{~km} \mathrm{~s}^{-1} \mathrm{Mpc}^{-1}, \Omega_{\mathrm{m}}=0.3$, and $\Omega_{\Lambda}=$ 0.7 . Error bars are given within a 68 per cent confidence interval. All the abundances mentioned in this work are given with respect to their proto-solar values obtained by Lodders, Palme \& Gail (2009).

\section{REANALYSIS OF THE CHEERS SAMPLE}

The sample, data reduction, background modelling, and spectral fitting strategy are all described in detail in Paper I (see also Mernier et al. 2015). Compared to our previous work, we discard the observation of M 89 (ObsID: 0141570101) because of its high background contamination. This leaves us with XMM-Newton EPIC observations of 43 nearby cool-core clusters, groups, and ellipticals, all being part of the CHEERS project (see also Pinto et al. 2015; de Plaa et al. 2017). The brightness of these nearby sources, combined to their relatively moderate temperature (not exceeding $\sim 8 \mathrm{keV}$ ), allows a robust determination of the $\mathrm{Fe}$ abundance with the EPIC instruments, based on the $\mathrm{Fe}-\mathrm{K}$ lines and/or the $\mathrm{Fe}-\mathrm{L}$ complex.

Unlike in Paper I, where the spectra were extracted within $0.05 r_{500}$ and/or $0.2 r_{500}$ (depending on the distance of the system), the goal of this paper is to measure the Fe abundance within the same physical scale. Therefore, all the spectra of our sample are reextracted and re-analysed within $0.1 r_{500}$. The only exception is the Virgo cluster (centred on M 87), which could be analysed only out to $0.05 r_{500}$ within the EPIC field of view. The redshift and hydrogen column density $\left(n_{\mathrm{H}}\right)$ values are adopted from Paper I.

\subsection{From SPEXACT v2 to SPEXACT v3}

A key improvement with respect to Paper I is the updated version of the SPEX Atomic Code and Tables (hereafter SPEXACT). While in Paper I our analysis relied on SPEXACT v2.05 (hereafter v2), in this Letter we take advantage of the up-to-date release of SPEXACT v3.04 (hereafter v3). This most recent version is the result of a major update started in 2016 (SPEXACT v3.00) with further minor improvements implemented until the end of 2017 (Hitomi Collaboration et al. 2017). Compared to SPEXACT v2, the total number of energy transitions has increased by a factor of $\sim 400$, to reach more than 1.8 million in SPEXACT v3. The new transitions include for instance higher principal quantum numbers for both $\mathrm{H}$-like and He-like ions. In addition, significant updates were performed in collisional excitation and de-excitation rates, radiative transition probabilities, auto-ionization, and dielectronic recombination rates (either from the literature or consistently calculated using the $\mathrm{FAC}^{2}$ code; $\mathrm{Gu}$ 2008). Finally, significant improvements were obtained in radiative recombination (Badnell 2006; Mao \& Kaastra 2016) and collisional ionization coefficients (Urdampilleta, Kaastra \& Mehdipour 2017). In order to compare the effects of the improvements in a consistent way, in the following we use successively SPEXACT v2 and SPEXACT v3 to fit all our EPIC spectra (MOS 1, MOS 2, and pn are fitted simultaneously, see Paper I).

\subsection{Multitemperature modelling}

As already demonstrated by e.g. Buote \& Canizares (1994), Buote (2000, Fe-bias), Rasia et al. (2008), and Simionescu et al. (2009, inverse Fe-bias), modelling the ICM with a multitemperature structure 


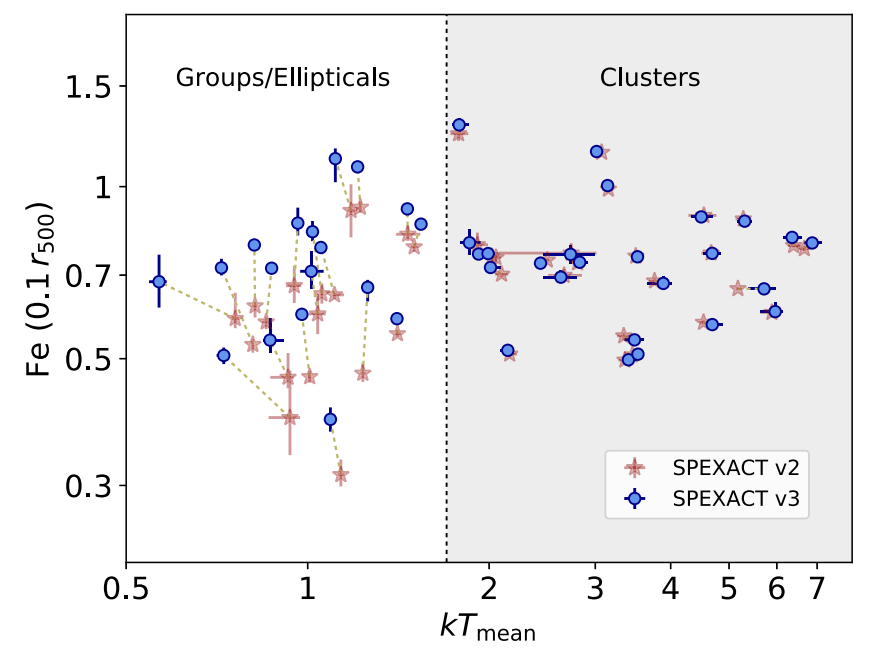

Figure 1. Iron abundance measured as a function of the mean temperature within $0.1 r_{500}$ of the ellipticals, galaxy groups, and clusters from the CHEERS sample (only M 89 is discarded, see the text). For a given system, the corresponding SPEXACT v2 (orange stars) and SPEXACT v3 (blue dots) measurements, both obtained using a 3T model (see the text), are tied by a green-brown dashed line. Clusters and groups/ellipticals are delimited arbitrarily beyond and below $k T_{\text {mean }}=1.7 \mathrm{keV}$, respectively.

is essential to derive correct abundances. The most intuitive assumption would be to consider that the temperature follows a Gaussian differential emission measure distribution (the gdem model; see e.g. de Plaa et al. 2006; Simionescu et al. 2009). Such a model, however, requires appreciable computing resources, especially when using SPEXACT v3. A cheaper, yet still reasonable alternative would be to approximate a gdem distribution by modelling three temperature components (3T): (i) the main component, for which the temperature $k T_{\text {mean }}$ and the emission measure $Y$ are left free in the fits; (ii) a higher- and (iii) a lower temperature components, whose temperatures $k T_{\text {up }}$ and $k T_{\text {low }}$ are left free but their $Y$ is tied to half of that of the main component. The ratio $k T_{\text {up }} / k T_{\text {low }}$ can thus be seen as the typical width of the distribution. Although such a temperature distribution may somewhat deviate from Gaussianity in some cases, we verify that fitting (i) a subsample of systems with a gdem model and (ii) gdem-simulated EPIC spectra with a 3T model across various mean temperatures have negligible impact (always less than $\sim 6$ per cent) on our measured Fe abundances.

Such multitemperature modelling is particularly relevant here, as we obtain significantly better fits than when we model our spectra with a single-temperature component only. Moreover, in addition to the fact that all our systems are classified as cool-core, they are also known to exhibit clear temperature gradients within $\sim 0.1 r_{500}$ (see e.g. results from the ACCEPT catalogue; Cavagnolo et al. 2009).

\section{RESULTS}

The measured Fe abundances of the 43 CHEERS systems reanalysed within $0.1 r_{500}$ are shown as a function of their $k T_{\text {mean }}$ in Fig. 1. Because the overall temperature of the ICM scales with the total mass $M$ of the system as $\sim M^{2 / 3}$ (e.g. Giodini et al. 2013), $k T_{\text {mean }}$ can be seen as a reasonable proxy for the total mass of our sources. Therefore, we split our sample into two subsamples, namely: (i) 'clusters', for which $k T_{\text {mean }}>1.7 \mathrm{keV}$, and (ii) 'groups/ellipticals', for which $k T_{\text {mean }}<1.7 \mathrm{keV}$. The choice of the threshold value $k T_{\text {mean }}=1.7 \mathrm{keV}$ is of course arbitrary, but well justified by the usual classification attributed to each system in the literature.

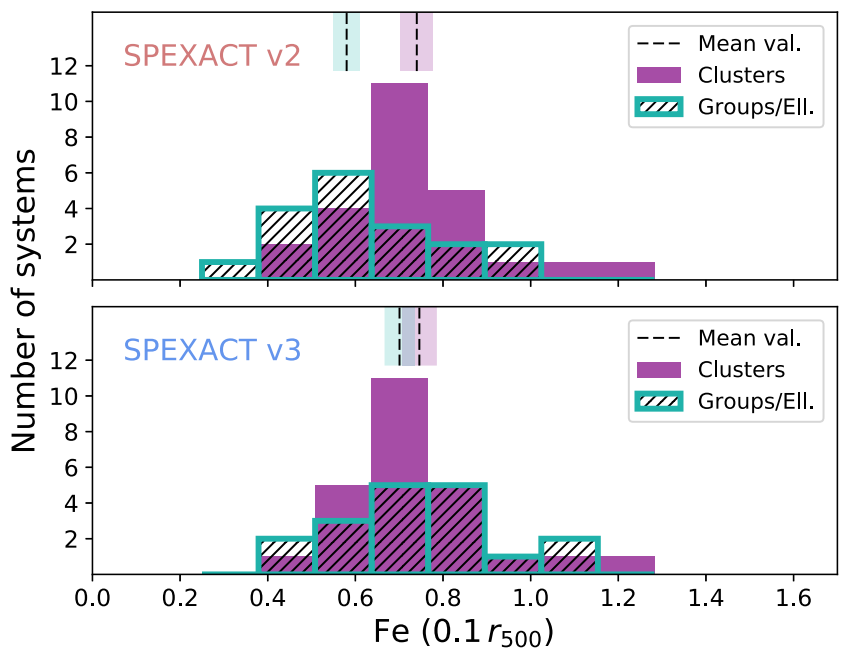

Figure 2. Histograms showing the $\mathrm{Fe}$ abundance distribution of the CHEERS sample, when using successively SPEXACT v2 (top panel) and SPEXACT v3 (bottom panel). In each case, the distribution for clusters $\left(k T_{\text {mean }}>1.7 \mathrm{keV}\right)$ and groups/ellipticals $\left(k T_{\text {mean }}<1.7 \mathrm{keV}\right)$ is shown separately. The mean value of each distribution (and corresponding errors) is shown by the vertical dashed lines (and filled areas around them).

While compared to SPEXACT v2, the Fe abundances measured in clusters remain essentially unchanged, the Fe abundances in groups and ellipticals are systematically revised upwards when using SPEXACT v3. This result is better quantified in Fig. 2, where the distribution of $\mathrm{Fe}$ abundances is compared between clusters and groups/ellipticals, using the two versions of the code. Based on the entire sample, the SPEXACT v3 results provide a mean Fe abundance of $0.74 \pm 0.03$ with an intrinsic scatter of 25 per cent (computed following the method described in Paper I). When splitting the sample, we find consistent average Fe abundances of $0.75 \pm 0.04$ and $0.70 \pm 0.03$ for clusters and groups/ellipticals, respectively. This is in contrast with the SPEXACT v2 results, where the average $\mathrm{Fe}$ abundance values for clusters $(0.75 \pm 0.04)$ and groups/ellipticals $(0.58 \pm 0.03)$ are significantly different. In other words, spectral fits obtained using updated atomic data indicate that the average concentration of $\mathrm{Fe}$ in the hot haloes of groups and giant ellipticals is the same as that in clusters of galaxies.

Systems for which $k T_{\text {mean }}$ lies within $2-3 \mathrm{keV}$ exhibit both Fe-L and $\mathrm{Fe}-\mathrm{K}$ lines, hence their $\mathrm{Fe}$ abundance can be constrained by each of these two features separately. We test this approach on M 87 and EXO 0422, both having good data quality. Compared to the $\mathrm{Fe}$ estimated from the 'full band' fits, we find that the $<2 \mathrm{keV}$ local fits ( $\mathrm{Fe}-\mathrm{L}$ lines only) provide negligible biases $(-2$ percent and +6 per cent for M 87 and EXO 0422, respectively). These biases become somewhat larger (respectively +15 per cent and -12 per cent) in the $>2 \mathrm{keV}$ local fits ( $\mathrm{Fe}-\mathrm{K}$ lines only); however they are not systematic and remain well below the typical 25 percent scatter reported above. Although this issue is well known (Rasia et al. 2008; Simionescu et al. 2009) and concerns less than $\sim 14$ per cent of our systems, this mismatch will deserve attention with future high-resolution spectroscopy missions.

\subsection{The code-related Fe-bias}

In clusters, the Fe abundance determination is predominantly based on the prominent $\mathrm{Fe}-\mathrm{K}$ lines. Since only the low-temperature groups/ellipticals are significantly affected by the update of SPEXACT, 


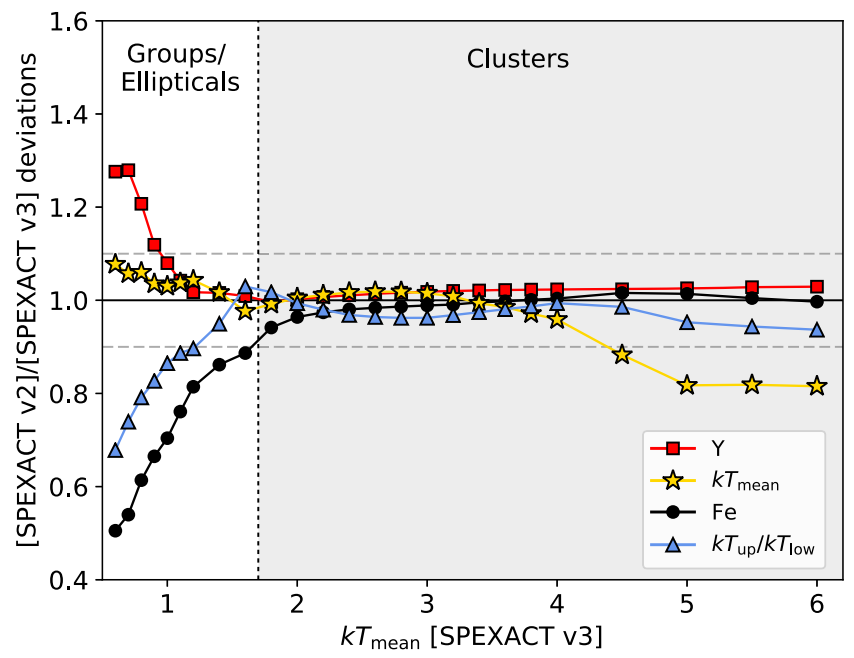

Figure 3. Relative deviations on the parameters $Y, k T_{\text {mean }}, \mathrm{Fe}$, and the ratio $k T_{\text {up }} / k T_{\text {low }}$ when EPIC mock spectra of 3T plasma (simulated using SPEXACT v3 for various initial mean temperatures) are fitted using SPEXACT v2. The two horizontal dashed lines indicate the \pm 10 per cent relative deviations.

the reason for such a change is to be found in the Fe-L emission, which is dominant at $k T_{\text {mean }} \lesssim 2 \mathrm{keV}$. In order to better understand the code-related Fe bias that we report above, we adapt an instructive exercise previously introduced in Mernier et al. (2017) and de Plaa et al. (2017). In short, we start by using SPEXACT v3 to simulate mock EPIC spectra with $100 \mathrm{ks}$ exposure on a grid of various $k T_{\text {mean }}$ values. In all these simulations $Y$ and the abundances are assumed to be $10^{72} \mathrm{~m}^{-3}$ and 1 proto-solar, respectively. Moreover, $k T_{\text {up }}$ and $k T_{\text {low }}$ are assumed such that $k T_{\text {up }} / k T_{\text {low }}=2.8$. As a second step, we fit these mock spectra using SPEXACT v2 with $Y, \mathrm{Fe}$, $k T_{\text {mean }}, k T_{\text {up }}$, and $k T_{\text {low }}$ as free parameters. The relative deviation of these SPEXACT v2 best-fitting parameters with respect to their input SPEXACT v3 values is shown in Fig. 3 as a function of the input mean temperature. As expected from our results above, the Fe consistency between the two versions of SPEXACT is excellent in the clusters regime, while it dramatically deteriorates when the plasma becomes cooler than $\sim 2 \mathrm{keV}$. In addition, other interesting effects occur in the groups/ellipticals regime. Below $k T_{\text {mean }} \lesssim 1.5 \mathrm{keV}$ and $k T_{\text {mean }} \lesssim 1 \mathrm{keV}$, the ratio $k T_{\text {up }} / k T_{\text {low }}$ and $Y$ are respectively underand overestimated by SPEXACT v2. The mean temperature, however, remains reasonably reproduced by SPEXACT v2, except for very hot plasmas where $k T_{\text {mean }}$ is at most $\sim 15$ per cent underestimated (though without affecting the Fe abundance).

To better understand all the biases we observe in cool plasmas with the EPIC instruments, we investigate further the case of a 3T plasma simulated for $100 \mathrm{ks}$ with SPEXACT v3, assuming $k T_{\text {mean }}=0.7 \mathrm{keV}$ (Fig. 4, black data points). A direct comparison of this simulated spectrum with its equivalent model using SPEXACT v2 (Fig. 4, red line) shows significant discrepancies throughout the entire Fe-L complex (0.6-1.2 keV). In fact, the emissivity of many important lines (e.g. Fe XVII at $\sim 0.73 \mathrm{keV}$; Fe XVIII at $\sim 0.77 \mathrm{keV}$ ) were revised lower with the update of SPEXACT, while new transitions were incorporated and/or updated with a higher emissivity (e.g. Fe XVIII at $\sim 1.18 \mathrm{keV}$ ). When fixing the Fe abundance to its best-fitting value estimated a posteriori by SPEXACT v2 (Fig. 4, orange line), the emitting bump at $\sim 0.7 \mathrm{keV}$ gets smoother, in better agreement with the overall shape of the Fe-L complex. However, over the entire soft band the flux significantly decreases, which the fit attempts to 'correct' by increasing $Y$ (Fig. 4, green line). Finally, the

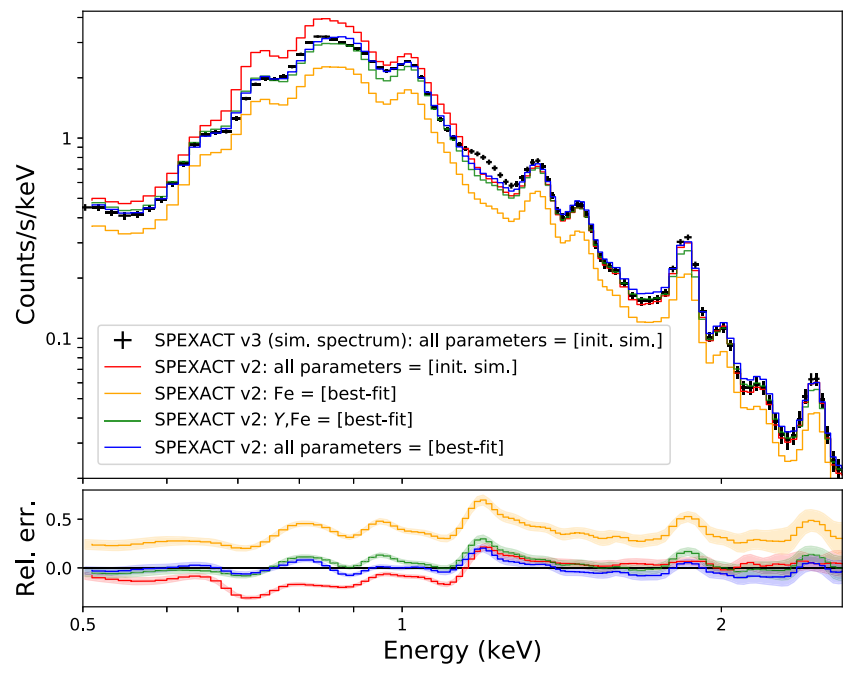

Figure 4. EPIC MOS 2 simulated spectrum of a $3 \mathrm{~T}$ plasma with $k T_{\text {mean }}=0.7 \mathrm{keV}$, using SPEXACT v3. For comparison, we show the same model calculated using SPEXACT v2 (red). Then, we progressively fix the Fe (orange), $Y$ (green), and eventually $k T_{\text {mean }}, k T_{\text {up }}$, and $k T_{\text {low }}$ (blue) to their a posteriori best-fitting SPEXACT v2 values. The residuals of such models with respect to the input simulated spectrum are shown in the bottom panel.

fit smooths the residual bumps (in particular around $\sim 0.9-1 \mathrm{keV}$ ) by simultaneously decreasing $k T_{\text {up }}$ and increasing $k T_{\text {low }}$ to provide a formally acceptable - but incorrect - best fit to the input spectrum (Fig. 4, blue line).

In summary, in cool plasmas the emission measure, the Fe abundance, and the width of the temperature distribution $\left(k T_{\text {up }} / k T_{\text {low }}\right)$ influence each other to reproduce the observed shape of the unresolved $\mathrm{Fe}-\mathrm{L}$ complex. As a consequence, even outdated spectral codes can reasonably fit the Fe-L complex, yet providing strongly biased measurements. This conspiracy between all these parameters explains the code-related Fe-bias that we report in this Letter.

\section{IMPLICATIONS FOR THE IRON CONTENT IN ELLIPTICALS, GROUPS, AND CLUSTERS}

By measuring Fe abundances within $0.1 r_{500}$ in a self-consistent way and using the latest SPEXACT version available to date, we report for the first time similar Fe abundances in ellipticals, galaxy groups, and galaxy clusters. In other words, gas-phase metallicities remain constant across two orders of magnitude in halo mass.

These new results contradict previous papers (e.g. Rasmussen \& Ponman 2009; Bregman et al. 2010; Sun 2012; Yates et al. 2017), which reported systematically lower Fe abundances in groups and/or ellipticals with respect to the hotter clusters of galaxies [although Konami et al. (2014) reported similar average Fe abundances as reported here, albeit for ellipticals only]. Rather than SPEX, most of those previous studies used many (very different) versions of APEC to fit their data, making a direct comparison with this work difficult. All these (mostly outdated) atomic codes, however, likely encountered similar problems of a too simplistic modelling of the $\mathrm{Fe}-\mathrm{L}$ transitions. From a theoretical perspective, that trend was not trivial to explain. For example, when comparing the observational trend with a semi-analytic model, Yates et al. (2017) did not succeed to reproduce the previously reported positive temperature-metallicity correlation in galaxy groups. Instead, the metal content in low-mass systems is systematically overestimated by their model. 
Our present results have interesting consequences, in particular given that the investigated systems exhibit very different stellar- to ICM-mass fractions. Because this fraction is lower in rich clusters than in less massive systems, invariant $\mathrm{Fe}$ abundances could be explained only if the effective ICM enrichment considerably increases with the mass of the system. Such requirements have been difficult to reconcile with the observed stellar populations in clusters so far (e.g. Loewenstein 2013; Renzini \& Andreon 2014). The story, however, is different if the Fe present in the ICM is unrelated to the current stellar population of these systems. In addition to the increasing evidence towards an early ICM enrichment in cluster outskirts (e.g. Werner et al. 2013; Simionescu et al. 2017; Urban et al. 2017), central Fe peaks were also found to be in place already at $z \sim 1$ (De Grandi et al. 2014; Mantz et al. 2017) and exhibit the same radial distribution as SNcc products (Mernier et al. 2017). These recent findings suggest that recent SNIa explosions and stellar mass-loss from central galaxies do not significantly contribute to the ICM enrichment.

In this context, the similar Fe abundances found in hot haloes spanning different mass ranges constitute an additional support towards this early enrichment scenario, even in their central parts. Since they grow hierarchically, isolated massive ellipticals and assembling groups can be seen as the first steps of the formation of more massive clusters. Although, admittedly, nearby groups may have different specific properties (star formation, active galactic nucleus feedback, etc.) than high-redshift proto-clusters, the massinvariance of $\mathrm{Fe}$ abundances at low redshift suggests that the bulk of metals in hot haloes was already in place well before clusters effectively assembled. Our new measurements are directly confronted to (and are found to be in good agreement with) recent chemo- and hydrodynamical simulations in a companion paper (Truong et al. 2018), to which we refer the reader for a more detailed discussion.

We remind that these integrated measurements cover $0.1 r_{500}$, without further information on their inner or outer spatial distributions. The question of whether clusters and groups/ellipticals are really self-similar in terms of metal enrichment would require at least to derive the individual abundance profiles for the entire sample using SPEXACT v3 (for a similar work using SPEXACT v2, see Mernier et al. 2017). Because of the non-negligible time required by SPEXACT v3 to fit each spectrum, we leave such a study for future work.

In addition to the code-related Fe bias discussed in this work, we also note from Fig. 3 that fitting the spectra of cool systems with an outdated plasma code may also bias the emission measure, the mean temperature and the $k T_{\text {up }} / k T_{\text {low }}$ ratio by +35 per cent, +7 per cent, and -24 per cent, respectively. In turn, these biases may have consequences on the estimates of further interesting quantities. For instance, we estimate that the ICM pressure, usually defined as $P=n_{\mathrm{e}} k T$, can be biased high by $\sim 19$ percent in the case of a $\sim 0.7 \mathrm{keV}$ plasma. Unlike the pressure, the ICM entropy, usually defined as $K=k T / n_{\mathrm{e}}^{2 / 3}$, remains very close to its true value, with a underestimate of less than $\sim 1$ per cent. Similarly, the total hydrostatic mass is not expected to be affected by more than a few per cent, as temperature and density gradients do not change dramatically. A more precise quantification, however, is left to future work. Our results also reveal the complication of measuring accurately the temperature structure of lower mass systems, as long as the Fe- $\mathrm{L}$ complex remains unresolved by the observing instruments.

Finally, it should be reminded that no spectral code is perfect. It is certain that further improvements on SPEXACT will be pursued in the future, with potential implications on the interpretation of moderate resolution spectra of X-ray sources. In that respect, micro- calorimeters onboard future missions such as XARM and Athena will enable us to observe the $\mathrm{Fe}-\mathrm{L}$ complex with unprecedented resolution. These observations will be invaluable to better understand all the radiation processes in the ICM and push our knowledge of astrophysical plasma emission to the next level.

\section{ACKNOWLEDGEMENTS}

The authors thank the referee for their constructive comments that helped to improve the manuscript as well as Kiran Lakhchaura for fruitful discussions. FM is supported by the Lendület LP2016-11 grant awarded by the Hungarian Academy of Sciences. This work is partly based on the XMM-Newton AO-12 proposal 'The XMMNewton view of chemical enrichment in bright galaxy clusters and groups' (PI: de Plaa), and is a follow-up of the CHEERS (CHEmical Evolution Rgs cluster Sample) collaboration; the authors thank all its members. This work is based on observations obtained with $X M M-N e w t o n$, an ESA science mission with instruments and contributions directly funded by ESA member states and the USA (NASA). The SRON Netherlands Institute for Space Research is supported financially by NWO, the Netherlands Organization for Scientific Research.

\section{REFERENCES}

Badnell N. R., 2006, ApJS, 167, 334

Biffi V., Planelles S., Borgani S., Rasia E., Murante G., Fabjan D., Gaspari M., 2018, MNRAS, 476, 2689

Biffi V. et al., 2017, MNRAS, 468, 531

Bregman J. N., Anderson M. E., Dai X., 2010, ApJ, 716, L63

Buote D. A., 2000, MNRAS, 311, 176

Buote D. A., Canizares C. R., 1994, ApJ, 427, 86

Cavagnolo K. W., Donahue M., Voit G. M., Sun M., 2009, ApJS, 182, 12

De Grandi S., Molendi S., 2001, ApJ, 551, 153

De Grandi S., Molendi S., 2009, A\&A, 508, 565

De Grandi S., Santos J. S., Nonino M., Molendi S., Tozzi P., Rossetti M., Fritz A., Rosati P., 2014, A\&A, 567, A102

de Plaa J., 2013, Astron. Nachr., 334, 416

de Plaa J., Mernier F., 2017, Astron. Nachr., 338, 299

de Plaa J., Werner N., Bleeker J. A. M., Vink J., Kaastra J. S., Méndez M., 2007, A\&A, 465, 345

de Plaa J. et al., 2006, A\&A, 452, 397

de Plaa J. et al., 2017, A\&A, 607, A98

Finoguenov A., Davis D. S., Zimer M., Mulchaey J. S., 2006, ApJ, 646, 143

Giodini S., Lovisari L., Pointecouteau E., Ettori S., Reiprich T. H., Hoekstra H., 2013, Space Sci. Rev., 177, 247

Giodini S. et al., 2009, ApJ, 703, 982

Grange Y. G., de Plaa J., Kaastra J. S., Werner N., Verbunt F., Paerels F., de Vries C. P., 2011, A\&A, 531, A15

Gu M. F., 2008, Can. J. Phys., 86, 675

Hitomi Collaboration et al., 2018, PASJ, 70, 12

Kaastra J. S., Mewe R., Nieuwenhuijzen H., 1996, in Yamashita K., Watanabe T., eds, UV and X-ray Spectroscopy of Astrophysical and Laboratory Plasmas, Universal Academy Press, Inc., Tokyo, Japan, p. 411

Konami S., Matsushita K., Nagino R., Tamagawa T., 2014, ApJ, 783, 8

Lodders K., Palme H., Gail H.-P., 2009, Landolt Börnstein, p. 44

Loewenstein M., 2013, ApJ, 773, 52

Mahdavi A., Finoguenov A., Böhringer H., Geller M. J., Henry J. P., 2005, ApJ, 622, 187

Mantz A. B., Allen S. W., Morris R. G., Simionescu A., Urban O., Werner N., Zhuravleva I., 2017, MNRAS, 472, 2877

Mao J., Kaastra J., 2016, A\&A, 587, A84

Matsushita K., 2011, A\&A, 527, A134

Mernier F., de Plaa J., Lovisari L., Pinto C., Zhang Y.-Y., Kaastra J. S., Werner N., Simionescu A., 2015, A\&A, 575, A37 
Mernier F., de Plaa J., Pinto C., Kaastra J. S., Kosec P., Zhang Y.-Y., Mao J., Werner N., 2016, A\&A, 592, A157 (Paper I)

Mernier F. et al., 2017, A\&A, 603, A80

Pinto C. et al., 2015, A\&A, 575, A38

Rasia E., Mazzotta P., Bourdin H., Borgani S., Tornatore L., Ettori S., Dolag K., Moscardini L., 2008, ApJ, 674, 728

Rasmussen J., Ponman T. J., 2007, MNRAS, 380, 1554

Rasmussen J., Ponman T. J., 2009, MNRAS, 399, 239

Renzini A., Andreon S., 2014, MNRAS, 444, 3581

Sasaki T., Matsushita K., Sato K., 2014, ApJ, 781, 36

Simionescu A., Werner N., Böhringer H., Kaastra J. S., Finoguenov A., Brüggen M., Nulsen P. E. J., 2009, A\&A, 493, 409

Simionescu A., Werner N., Mantz A., Allen S. W., Urban O., 2017, MNRAS, 469,1476

Sun M., 2012, New J. Phys., 14, 045004

Tamura T., Kaastra J. S., den Herder J. W. A., Bleeker J. A. M., Peterson J. R., 2004, A\&A, 420, 135

Truong N. et al., 2018, preprint (arXiv:1803.06297)

Urban O., Werner N., Allen S. W., Simionescu A., Mantz A., 2017, MNRAS, 470,4583
Urdampilleta I., Kaastra J. S., Mehdipour M., 2017, A\&A, 601, A85

Werner N., Durret F., Ohashi T., Schindler S., Wiersma R. P. C., 2008, Space Sci. Rev., 134, 337

Werner N., Urban O., Simionescu A., Allen S. W., 2013, Nature, 502, 656

Yates R. M., Thomas P. A., Henriques B. M. B., 2017, MNRAS, 464, 3169

Zhang Y.-Y., Laganá T. F., Pierini D., Puchwein E., Schneider P., Reiprich T. H., 2011, A\&A, 535, A78

\section{SUPPORTING INFORMATION}

Supplementary data are available at MNRASL online.

Please note: Oxford University Press is not responsible for the content or functionality of any supporting materials supplied by the authors. Any queries (other than missing material) should be directed to the corresponding author for the article.

This paper has been typeset from a $\mathrm{T}_{\mathrm{E}} \mathrm{X} / \mathrm{L} \mathrm{T} \mathrm{E} \mathrm{X}$ file prepared by the author. 